\title{
LA JORNADA MUNDIAL DE LA JUVENTUD Y SUS REPERCUSIONES EN LAS EMPRESAS HOTELERAS DE LA CIUDAD DE PANAMÁ
}

The world youth day and its repercussion in the hotel companies of Panama city

\author{
Nelly Araya \\ Profesora, Facultad de Administración de Empresas y Contabilidad, Universidad de Panamá, \\ narraya16@yahoo.com
}

Resumen

\begin{abstract}
Dentro del contexto del turismo religioso, modalidad motivada por el deseo asistir a eventos o conocer destinos religiosos, en enero del 2019 se celebró en la ciudad de Panamá la Jornada Mundial de La Juventud (JMJ). Este evento, realizado cada tres años, se llevó a cabo entre los días 22 y 27, y de acuerdo con la información suministrada por el sitio oficial de la JMJ Panamá 2019, reunió más de 700.000 personas. Con un evento de tal magnitud, surgen las interrogantes sobre los impactos en los diferentes sectores del país, esta interrogante es tratada en esta investigación exploratoria documental con respecto, específicamente al sector hotelero de Ciudad de Panamá. Se contrastaron las notas de prensa encontradas sobre el tema con documentos oficiales de la Organización Mundial del Turismo y artículos de investigación en otros casos exitosos de turismo religioso en el ámbito iberoamericano. El artículo concluye sobre los efectos positivos en lo económico y mercadotécnico para el sector hotelero pero también subraya la necesidad de sistematizar la información requerida y la puesta en marcha de sistemas de inteligencia de mercado turístico y la cuenta satélite.
\end{abstract}

Palabras claves: Jornada Mundial, Panamá, Turismo religioso, Sector Hotelero, economía.

\begin{abstract}
Within the context of religious tourism, a modality motivated by the desire to attend events or know religious destinations, the World Youth Day (WYD) was celebrated in Panama City in January 2019. This event, held every three years, took place between 22 and 27, and according to information provided by the official site of WYD Panama 2019, gathered more than 700,000 people. With an event of such magnitude, questions arise about the impacts on different sectors of the country, this question is addressed in this exploratory documentary research with respect to the hotel sector of Panama City. The press releases found on the subject were contrasted with official documents of the World Tourism Organization and research articles on other successful cases of religious tourism in the Ibero-American area. The article concludes on the positive economic and marketing effects for the hotel sector but also underlines the need to systematize the required information and the implementation of tourist market intelligence systems and the satellite account.
\end{abstract}

Keywords: World Youth, Panama, religious tourism, hotel sectors, economy. 


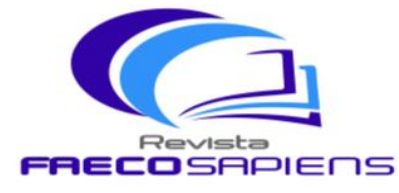

Revista ค月EடロSคคIEחS
ताT Volumen 3 - Número 1

Enero-Junio 2020

Recibido: 21/10/19 Aceptado: 20/11/19 pp. 41-52 Indexada

\section{INTRODUCCIÓN}

El 23 de mayo de 2019, el Papa Francisco recibió en el Vaticano al Secretario General de la Organización Mundial del Turismo (OMT), Zurab Pololikashvili, al terminar la reunión, este último expresó: "El turismo y la religión tienen mucho en común. Ambos se esfuerzan por hacer del mundo un lugar mejor mediante la erradicación de la pobreza y la promoción de la paz." (UNWTO, 2019).

En efecto, el turismo religioso, tiene un impacto positivo en las comunidades receptivas en la medida en que su planificación y ejecución, están dentro de los criterios que la misma OMT establece como visión de turismo sostenible e inclusivo, de tal manera, esta modalidad tiene reconocimiento por la máxima organización turística mundial en cuanto al impacto que genera, social, patrimonial y económicamente, como la mayoría, por no decir todas, las modalidades turísticas. El turismo religioso puede ser motivado por el deseo de conocer destinos de connotación religiosa, cualquiera que esta sea, y los atractivos de corte patrimonial que se encuentren en ella, así, casos como la Meca o Santiago de Compostela, son icónicos, por otro lado se encuentra la motivación surgida por el deseo de participar en algún evento de corte religioso, es el caso de la Jornada Mundial de La Juventud (JMJ).

Este evento, que se realiza cada tres años, en esta oportunidad tuvo como locación a Panamá, entre el 22 y el 27 de Enero de este año, y de acuerdo con la información suministrada por el sitio oficial de la JMJ Panamá 2019, en la misa conclusiva se congregaron más de 700.000 personas (Jornada Mundial de Juventud Panamá 2019, 2019).

Un evento de tal magnitud, debe tener impactos en los diferentes sectores del país, de acuerdo con lo que más arriba se comentó por la OMT, en efecto, el evento generó 250 millones de dólares según afirmó, Gustavo Him, administrador de la Autoridad de Turismo de Panamá (Panamá América, 2019), ¿pero qué significa eso? ¿Qué tipos de externalidades se han reconocido y cuál ha sido su magnitud? Están interrogantes salen inevitablemente a la luz, y en esta investigación se trata de responder a una de ellas: ¿Qué repercusiones trajo la JMJ Panamá 2019 para las empresas hoteleras de Ciudad de Panamá? 


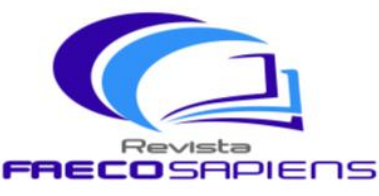

ताT Volumen 3 - Número 1 Enero-Junio 2020

Recibido: 21/10/19 Aceptado: 20/11/19 pp. 41-52 Indexada

Con el objeto de responder a esta interrogante, se planteó una investigación exploratoria de tipo documental donde se revisaron las notas de prensa que al respecto se han emitido, a la vez de teoría y experiencias en otros países publicadas sobre los efectos del turismo religioso.

\section{CONTENIDO}

Se revisaron los artículos y notas de prensa más relevantes encontrados en la web sobre los impactos de la JMJ en las empresas hoteleras en Ciudad de Panamá o que pudieran relacionarse, se sistematizó la información rescatándose la información común proveniente de fuentes autorizadas en notas de prensa siendo que hasta el momento no se han encontrado indicadores estadísticos oficiales y se comparó con la teoría y casos exitosos de turismo religioso en el ámbito iberoamericano.

La World Religious Travel Association (WRTA) definió al turismo religioso como "...un viaje turístico donde el elemento religioso constituye uno de los objetivos principales". ( Tobón Perilla \& Tobón Perilla, 2013, pág. 238) y, de acuerdo a la OMT (2007) las tres dimensiones fundamentales de esta modalidad son:

- Las peregrinaciones a destinos, con una característica predominantemente turística.

- Los encuentros y eventos religiosos y espirituales, como es el caso de la JMJ.

- Los itinerarios y caminos a lugares de peregrinación en espacios territorios y espacios rurales.

Del mismo informe, emitido en ocasión de la Conferencia de Córdoba, entre el 29 y el 31 de octubre de 2007 (OMT, 2007), se pueden extraer los beneficios que este tipo de turismo plantea para los destinos:

- Estimula el diálogo entre civilizaciones.

- Fomenta la salvaguarda del patrimonio.

- Promueven la notoriedad de las antiguas peregrinaciones sus rutas e itinerarios religiosos. 


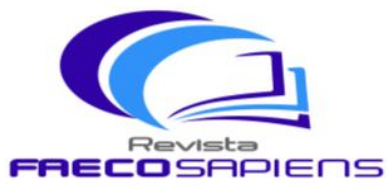

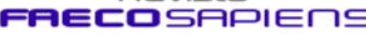

TाT Volumen 3 - Número 1

Enero-Junio 2020

Recibido: 21/10/19 Aceptado: 20/11/19 pp. 41-52 Indexada

- Estimulan la gestión y promoción eficientes de los destinos; uso de intensivo de las tecnologías de la información y de la comunicación (TIC) y protección de los recursos y entorno natural y cultural.

- Transforma los caminos de peregrinación y los itinerarios religiosos en productos turísticos permitiendo la ordenación del territorio y el desarrollo de las comunidades que atraviesan.

- Propicia el aumento y creación de agencias de viajes y turismo especializadas hacia el turismo religioso y espiritual y las TIC.

- Favorece el crecimiento y aparición del alojamiento de peregrinos y visitantes, diversificando la oferta, desde albergues a hotelería de lujo.

- Amplía el poder de atracción turística y sus derrames positivos como ingresos y empleos.

- Estimula las asociaciones entre el turismo y sectores como la agricultura o la artesanía.

De acuerdo a la Arquidiócesis de Panamá, la JMJ es un evento religioso que se propone como objetivo: “...dar a conocer a los jóvenes de hoy en día el mensaje de Cristo, y crear un ámbito abierto y de convivencia para compartir y reflexionar juntos sobre los temas fundamentales de la existencia." (Arquidiócesis De Panamá, S.F). Continúa el mismo sitio, explicando la historia y otras características de la JMJ, organizado por la Iglesia Católica, el cual es efectuado durante una semana cada tres años y presidido por el Papa; la idea data del Jubileo de 1983 - 1984, en Roma y en el siguiente año, en el marco del Año Internacional de la Juventud, proclamado por la ONU, el Papa Juan Pablo II instituyó la JMJ. (Arquidiócesis De Panamá, S.F).

El martes con la Eucaristía comienza la jornada con el Obispo de la localidad, llegando el Papa el jueves, el evento consiste en catequesis en las mañanas y eventos culturales y musicales en las tardes, siempre asociados a la fe y el cristianismo, en la tarde el viernes usualmente se representa el Vía Crucis, el sábado se dedica a vigilia de oración con el Papa, culminando el evento con la Misa final el domingo. (Arquidiócesis De Panamá, S.F)

- Contexto hotelero Panameño

Al tratar de establecer las condiciones en que se encuentra la hotelería en Ciudad de 


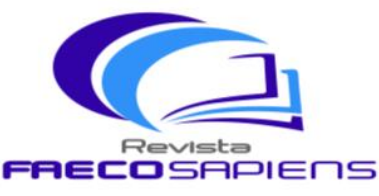

शTा Volumen 3 - Número 1 DRJI Enero-Junio 2020

Recibido: 21/10/19 Aceptado: 20/11/19 pp. 41-52 Indexada

Panamá, lo primero que surge es la escasa información publicada, así, la Autoridad de Turismo de Panamá, en base a datos otorgados por los hoteles de la Ciudad de Panamá, reportó un $47.1 \%$ de ocupación global entre enero y diciembre de 2017 , y de $44.9 \%$ en el mismo período en el año 2018 (Autoridad de Turismo de Panamá, 2019) esto, con alguna diferencia, es consistente con otras estadísticas oficiales (República de Panamá. Contraloría General), como se aprecia en la siguiente tabla:

Tabla1. Indicadores de ocupación hotelera Panamá. Período Enero - Agosto

\begin{tabular}{|l|l|l|l|l|}
\hline Año & $\begin{array}{c}\text { Unidades de } \\
\text { habitación al } \\
\text { final del período }\end{array}$ & $\begin{array}{c}\text { Promedio de } \\
\text { cuartos } \\
\text { ocupados }\end{array}$ & \multicolumn{1}{|c|}{$\begin{array}{c}\text { Promedio de } \\
\text { pernoctaciones }\end{array}$} & $\begin{array}{c}\text { Porcentaje de } \\
\text { ocupación }\end{array}$ \\
\hline 2016 & 10,449 & 5,054 & 10,871 & 48.3 \\
\hline 2017 & 10,500 & 5,004 & 13,410 & 47.8 \\
\hline 2018 & 10,205 & 4,672 & 13,753 & 45.4 \\
\hline
\end{tabular}

Fuente: Elaboración propia, en base a cifras de la Contraloría General de la República de Panamá.

A estas cifras, el sector hotelero ha respondido en forma crítica, así, en el artículo Un desesperado sector hotelero panameño busca un alivio económico con la JMJ (Sánchez, 2018), el presidente de la asociación de hoteles locales, Armando Rodríguez, reportó que más de 25.000 empleos se habían perdido para el momento, expresando que la situación era debida a la falta de promoción internacional entre 2010 y 2016, por otro lado, el directivo cifraba esperanzas en la Jornada Mundial de la Juventud en el 2019, expresando que contaban con “...30.000 cuartos de hospedaje en el país para albergar a los visitantes, aunque muchos de los jóvenes peregrinos se alojarán en casas y escuelas". (Sánchez, 2018).

La revisión de la red arrojó información que se puede sintetizar en la siguiente tabla de notas de prensa, debido a que en general es la que se encuentra repetida o comúnmente en los principales medios digitales del país. 
REVISTA FAECO SAPIENS

ISSN L 2644-3821

Acceso Abierto. Disponible en:

https://revistas.up.ac.pa/index.php/faeco_sapiens
TाV Volumen 3 - Número 1

Enero-Junio 2020

Recibido: 21/10/19 Aceptado: 20/11/19 pp. 41-52 Indexada

Tabla 2. Síntesis de hallazgos en relación a los efectos de la JMJ en los hoteles de Ciudad Panamá.

\begin{tabular}{|c|c|}
\hline Documento & Información aportada \\
\hline $\begin{array}{l}\text { Iberia firma } \\
\text { alianza para } \\
\text { promover turismo } \\
\text { en Panamá } \\
\text { (Hosteltur. } \\
\text { Edición Latam. } \\
\text { Marketing y } \\
\text { conectividad, } \\
\text { 2019) }\end{array}$ & $\begin{array}{l}\text { - El presidente de la junta directiva del Fondo de Promoción } \\
\text { Turística de Panamá, Fernando Duque resaltó la crisis del } \\
\text { turismo debido a la falta de publicidad internacional. } \\
\text { - La Autoridad de Turismo de Panamá (ATP), informó que en } \\
2018 \text { arribaron } 2,4 \text { millones de turistas al país, un descenso de } \\
1,4 \% \text { en relación al } 2017 \text {, pero el gasto aumentó } 3,3 \% \\
\text { alcanzando } 4.605 \text { millones de dólares. }\end{array}$ \\
\hline $\begin{array}{l}\text { JMJ impacta de } \\
\text { manera positiva } \\
\text { la imagen } \\
\text { turística de } \\
\text { Panamá (Villar, } \\
2019 \text { ) }\end{array}$ & $\begin{array}{l}\text { - Gustavo Him, administrador general de la Autoridad de Turismo } \\
\text { de Panamá (ATP) comunicó que en enero de } 2019 \text { con JMJ se } \\
\text { habían contabilizado un promedio de } 265,506 \text { visitantes, con un } \\
\text { impacto mediático personificado en } 2,500 \text { periodistas de todo el } \\
\text { mundo, y la exposición por más de } 60,000 \text { peregrinos } \\
\text { extranjeros en redes sociales, significando al turismo como "el } \\
\text { gran ganador". } \\
\text { Paulo Rezende, director comercial de Amadeus en Suramérica } \\
\text { anunció que la JMJ "contribuyó con un aumento del } 60 \% \text { en la } \\
\text { cantidad de reservas" de Panamá en relación al mismo mes el } \\
\text { año pasado, según datos obtenidos a partir de Amadeus } \\
\text { Destination Insight, herramienta de Big Data para destinos } \\
\text { turísticos, principalmente Brasil (298\%) México (207\%), Perú } \\
\text { (74\%), Colombia (45\%) y Estados Unidos (32\%) y Alemania, } \\
\text { con búsquedas sobre Panamá de 158\% superiores en relación } \\
\text { con enero de } 2018 \text {, aun cuando no fue de los países con } \\
\text { mayores concreciones. } \\
\text { Domingo de Obaldía, gerente general del restaurante El } \\
\text { Trapiche y presidente de la Asociación de Restaurantes y }\end{array}$ \\
\hline
\end{tabular}




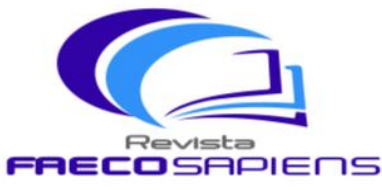

ஈคEடロதคค|กร
गाTे Volumen 3 - Número 1

Enero-Junio 2020

Recibido: 21/10/19 Aceptado: 20/11/19 pp. 41-52 Indexada

\begin{tabular}{|c|c|}
\hline & $\begin{array}{l}\text { Afines de Panamá (ARAP), resaltó la diferencia en los impactos } \\
\text { recibidos de acuerdo a la ubicación de los establecimientos, por } \\
\text { ejemplo varios restaurantes situados en la Vía Argentina } \\
\text { tuvieron resultados favorables, no así, otros locales en el Casco } \\
\text { Antiguo, posiblemente por el perfil joven de los peregrinos, } \\
\text { alojados en casas de familia, esperando una mejoría para } \\
\text { finales de semana con un perfil adulto y mayor capacidad de } \\
\text { gasto. Por último recordó que el sector tuvo un descenso } \\
\text { aproximado de } 40 \% \text { en ventas en el } 2018 \text { “...y la expectativa, } \\
\text { con la JMJ, era recuperar al menos } 25 \% \text { de consumo." } \\
\text { Armando Rodríguez, presidente de la Asociación Panameña de } \\
\text { Hoteles (Apatel), manifestó que la ocupación debido a la JMJ } \\
\text { se mantuvo en el } 60 \% \text { estimado originalmente pero la tarifa } \\
\text { promedio aumentó } 30 \% \text {, con ocupaciones en habitaciones } \\
\text { superiores a los } \$ 100 \text {. }\end{array}$ \\
\hline $\begin{array}{l}\text { Jornada Mundial } \\
\text { de la Juventud de } \\
\text { Panamá 2019, la } \\
\text { más acogedora } \\
\text { (Samaniego, } \\
\text { 2019) }\end{array}$ & $\begin{array}{l}\text { - GAD3, una consultora de investigación social y comunicación } \\
\text { con sede en Madrid, notificó que en la JMJ Panamá } 2019 \text { el } \\
50 \% \text { de los peregrinos que llegaron al país se alojaron en casas } \\
\text { de familias de acogida, además que esta JMJ “...ha sido la } \\
\text { tercera más mediática en la prensa internacional de las } \\
\text { jornadas celebradas desde el año } 2000, \text { solo superada por la } \\
\text { Río de Janeiro, Brasil, en } 2013 \text {, y la de Cracovia tres años más } \\
\text { tarde” con } 30 \% \text { de las noticias en español. } 22 \% \text { en inglés; } 17 \% \\
\text { en portugués; } 15 \% \text { italiano; } 10 \% \text { alemán; y un } 6 \% \text { en idioma } \\
\text { francés. }\end{array}$ \\
\hline $\begin{array}{l}\text { JMJ dejó balance } \\
\text { mixto en 'malls', } \\
\text { restaurantes y } \\
\text { hoteles (Castillo, } \\
2019 \text { ) }\end{array}$ & $\begin{array}{l}\text { - Nadyi Duque, presidenta de la Asociación Panameña de } \\
\text { Centros Comerciales indicó sobre la actividad en los food court, } \\
\text { que sobrepasaron las expectativas originales, no así en los } \\
\text { comercios donde se contrajeron las ventas. } \\
\text { - El presidente de la Asociación Panameña de Hoteles } \\
\text { (APATEL), Armando Rodríguez anunció que se había esperado }\end{array}$ \\
\hline
\end{tabular}




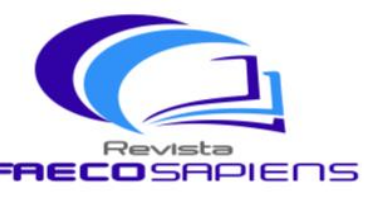

\begin{tabular}{|c|c|}
\hline & $\begin{array}{l}\text { una ocupación del 100\%, pero se alcanzó un } 60 \% \text {, } \\
\text { posiblemente por cancelaciones de grupos debido a } \\
\text { imposibilidad de acceso y transporte. } \\
\text { - Mientras, el presidente de ARAP aseveró que la llegada de } \\
\text { más peregrinos a Panamá, fue afectada fundamentalmente por } \\
\text { el alto costo en el pasaje aéreo. } \\
\text { - El administrador de la Autoridad de Turismo de Panamá (ATP), } \\
\text { Gustavo Him, certificó que la JMJ produjo alrededor } \$ 250 \\
\text { millones en ingresos económicos, con un ingreso por el } \\
\text { Aeropuerto Internacional de Tocumen de aproximadamente } 306 \\
\text { mil personas por la JMJ, excediendo las expectativas de por } \\
\text { más de } 50 \text { mil peregrinos, no obstante el director del Servicio } \\
\text { Nacional de Migración (SNM), Javier Carrillo, aclaró que sólo } \\
70 \text { mil } 803 \text { fueron peregrinos inscritos mientras que } 311 \text { mil } \\
679 \text { fueron viajeros. }\end{array}$ \\
\hline $\begin{array}{l}\text { Más de } 25 \text { mil } \\
\text { visitantes usaron } \\
\text { la APP de Uber } \\
\text { durante la JMJ } \\
\text { Panamá (Castillo, } \\
\text { 2019) }\end{array}$ & $\begin{array}{l}\text { - El Causeway, el Casco Viejo, Albrook Mall, y Multiplaza, fueron } \\
\text { los destinos más populares durante la JMJ. }\end{array}$ \\
\hline
\end{tabular}

Fuente: elaboración propia 


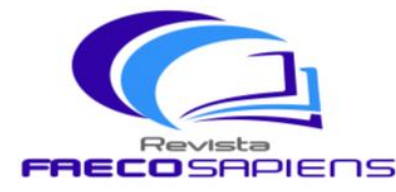

Revista

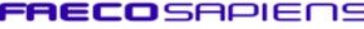

गाT Volumen 3 - Número 1

Enero-Junio 2020

Recibido: 21/10/19 Aceptado: 20/11/19 pp. 41-52 Indexada

\section{CONCLUSIONES}

Haciendo uso del concepto hermenéutico de la triangulación, se pueden emitir algunas opiniones comparando la teoría y la información disponible hasta el momento sobre los impactos de la JMJ 2019 en la hotelería de Ciudad Panamá:

- Lo primero que se concluye es que efectivamente la JMJ significó un aumento real de la ocupación hotelera, pero todavía no está claro si este aumento será sostenido, la caída posterior de las reservaciones y ventas parecieran poner en duda esto; siendo que la JMJ como evento, radica en un visitante que es leal a la jornada en sí misma, no al destino, no se espera una significativa tasa de retorno, sin embargo eso no debería ser una sorpresa, por cuanto la naturaleza de los arribos y reservaciones debía estar claro, la idea en todo caso es utilizar esta oportunidad para promocionar los servicios en aras de mantener vínculos con los visitantes y turistas llegados, es decir establecer programas de post venta, seguimiento al huésped y planes de fidelidad al cliente a fin de capitalizar esta visita a Panamá, que seguramente en muchos casos fue la primera.

- La visibilización y promoción del destino es notoria, las evidencias apuntan a una efectiva publicidad de las jornadas, no obstante haría falta más información en cuanto a alcance, impacto y profundidad de las campañas, esta publicidad es un efecto positivo que debe ser capitalizado mediante estrategias y campañas de mercadeo y promoción continua. Se requiere una estrategia promocional acordada entre los entes gubernamentales con competencia y los diferentes sectores privados, a fin de planificar y ejecutar un plan que cubra los requerimientos de todos los involucrados, aun cuando haya que priorizar en cuanto qué y cómo promocionar, debería tenerse en cuenta que en esta oportunidad hubo sectores de la hospitalidad que no recibieron en realidad los beneficios de la JMJ, esto debe y puede resolverse para futuros eventos mediante mayor publicidad en el destino, no solamente en los núcleos de origen de los turistas y visitantes, que obviamente no puede descuidarse.

- Se requiere una mejor planificación estratégica intersectorial, con el objetivo de mejorar y extender los beneficios de este tipo de actividad a otros sectores como alimentos y bebidas, artesanía, guías de turistas, etc. Ya se comentó la necesidad de revisar las estrategias promocionales, aquí de lo que se trata es de la operación 
REVISTA FAECO SAPIENS

ISSN L 2644-3821

Acceso Abierto. Disponible en:

https://revistas.up.ac.pa/index.php/faeco_sapiens

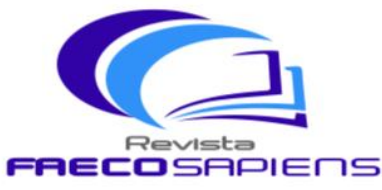

ताT Volumen 3 - Número 1

Enero-Junio 2020

Recibido: 21/10/19 Aceptado: 20/11/19 pp. 41-52

Indexada

receptiva, por ejemplo ampliar la oferta de los paquetes todo incluido, o al menos con visitas incluidas, estimular la creación de corredores turísticos como productos integrales, se debe recordar que la empresa hotelera es el servicio, es decir permite la permanencia del visitante en el destino, no es el atractivo, lo cual genera el desplazamiento, si bien un hotel, pudiera convertirse en sí mismo en un atractivo, en general lo ideal es que su capacidad de alojamiento sea potenciada por los recursos culturales y naturales del destino donde se encuentra, siendo así que la actividad turística puede generar los beneficios esperados dentro del marco de la sostenibilidad, entonces, con esta fórmula el hotel aumenta sus ingresos a la par de las demás empresas del sitio y la comunidad en general.

- Posiblemente la conclusión más relevante y con la cual finalizamos este artículo es la necesidad de sistematizar la información que este tipo de eventos debe generar, para eso las recomendaciones son:

- Culminar el proyecto de la cuenta satélite de turismo de Panamá, este sistema de información macroeconómica, suministra información que permite describir en profundidad la derrama del turismo en la economía del país, facilitando la identificación de las actividades económicas que producen los bienes y servicios reservados a los turistas; para el diseño y estructuración de esta cuenta deberían adoptarse recomendaciones internacionales como la Cuenta Satélite del Turismo de la OMT y el Sistema de Cuentas Nacionales.

- Elaborar investigaciones de mercado regulares, de alcance internacional, pero también nacional, e incentivar a las empresas y gremios a elaborar las suyas.

- Diseñar y aplicar un sistema de inteligencia de mercado turístico, que incluya el sistema de información turística y las investigaciones de mercado elaboradas, a fin de contar con data que sustente la toma de decisiones, la planificación y ejecución de estrategias sobre información oportuna, regular, confiable y disponible para todos los involucrados en la operación turística del país, y, para efectos de este artículo, para el sector hotelero en particular. 


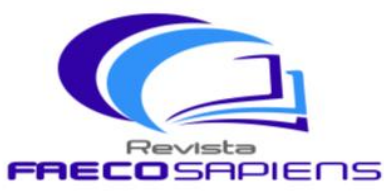

TाT Volumen 3 - Número 1

\section{REFERENCIAS BIBLIOGRÁFICAS}

Arquidiócesis De Panamá. (S.F). Arquidiócesis De Panamá. Obtenido de Jornada Mundial de la Juventud (JMJ): https://arquidiocesisdepanama.org/que-es-la-jmj/

Autoridad de Turismo de Panamá. (3 de Septiembre de 2019). Autoridad de Turismo de Panamá. Obtenido de Información de la ATP: https://www.atp.gob.pa/analisisdiagnostico-general-del-turismo-en-panama

Castillo (2019). JMJ dejó balance mixto en 'malls', restaurantes y hoteles. Panamá América. Recuperado el 15 de Octubre de 2019, de https://www.panamaamerica.com.pa/economia/jmj-dejo-balance-mixto-en-mallsrestaurantes-y-hoteles-1127185

Hosteltur. Edición Latam. Marketing y conectividad. (5 de Septiembre de 2019). Iberia firma alianza para promover turismo en Panamá. Hosteltur. Edición Latam. Marketing y conectividad. Recuperado el 30 de Septiembre de 2019, de https://www.hosteltur.com/lat/131025_iberia-firma-alianza-para-promover-turismo-enpanama.html

Jornada Mundial de Juventud Panamá 2019. (28 de Enero de 2019). JMJ Panamá 2019. Recuperado el 25 de Septiembre de 2019, de Un nuevo Panamá ha renacido con la JMJ 2019: https://saladeprensa.panama2019.pa/un-nuevo-panama-ha-renacido-conla-jmj-2019/

OMT. (2007). Turismo y Religiones. Cordova: OMT. Recuperado el 5 de Noviembre de 2019, de http://sdt.unwto.org/sites/all/files/pdf/summary_sp.pdf

Panamá América. (02 de Febrero de 2019). \$250 millones dejó la Jornada Mundial de la Juventud, según la ATP. Panamá América. Recuperado el 24 de Septiembre de 2019, de https://www.panamaamerica.com.pa/economia/250-millones-dejo-lajornada-mundial-de-la-juventud-segun-la-atp-1127097

República de Panamá. Contraloría General . (s.f.). PRINCIPALES INDICADORES ECONÓMICOS MENSUALES EN LA REPÚBLICA: ENERO-AGOSTO 2018-19. Instituto Nacional de Estadística y Censo. Obtenido de https://www.contraloria.gob.pa/inec/archivos/A4012019_hoteles.pdf

Samaniego, (2019). (Jornada Mundial de la Juventud de Panamá, la más acogedora de Enero de 2019). Jornada Mundial de la Juventud de Panamá 2019, la más acogedora. La Prensa/Panorama. Recuperado el 10 de Octubre de 2019, de https://impresa.prensa.com/panorama/Jornada-Mundial-Juventud-Panamaacogedora_0_5225477486.html 


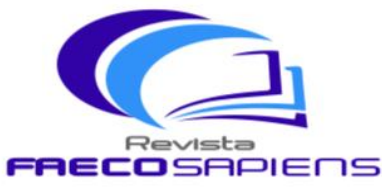

Volumen 3 - Número 1
Enero-Junio 2020 DRJI

Recibido: 21/10/19 Aceptado: 20/11/19 pp. 41-52 Indexada

la JMJ. EFE servicios. Recuperado el 10 de Octubre de 2019, de

https://www.efe.com/efe/america/sociedad/un-desesperado-sector-hoteleropanameno-busca-alivio-economico-con-la-jmj/20000013-3509116

Tobón (2013). TURISMO RELIGIOSO: FENóMENO SOCIAL Y ECONóMICO. Anuario Turismo y Sociedad, 14, 297 - 249. Recuperado el 22 de Septiembre de 2019, de http://search.ebscohost.com/login.aspx?direct=true\&db=fap\&AN=96047356\&lang=es \&site=ehost-live

UNWTO (2019). UNWTO. Recuperado el 25 de Septiembre de 2019, de El Secretario General de la OMT se reúne con el Papa Francisco para celebrar los valores compartidos de la religión y el turismo: http://www2.unwto.org/es/press-release/201905-23/el-secretario-general-de-la-omt-se-reune-con-el-papa-francisco-para-celebra

Villar, (2019). JMJ impacta de manera positiva la imagen turística de Panamá. El Capital Financiero.Com. Recuperado el 15 de Octubre de 2019, de https://elcapitalfinanciero.com/jmj-impacta-de-manera-positiva-la-imagen-turistica-depanama/ 